\title{
ON FUNCTIONS WITH A FINITE OR LOCALLY BOUNDED DIRICHLET INTEGRAL
}

\author{
MATTI VUORINEN
}

\section{Introduction}

The modulus of a curve family is an effective tool in the geometric function theory and in the theory of quasiconformal and quasiregular mappings (cf. [1], [13], [18], [19]). The aim of this paper is to show that the modulus method applies to the study of real-valued functions as well. We shall give an approach to the theory of Dirichlet finite functions, which is based on the well-known connection between the Dirichlet integral of an $\mathrm{ACL}^{n}$ function $u$ and the modulus of the family of curves joining given level sets of $u$. In this approach the modulus method has a role similar to that of the length-area principle in [7] and [17]. We shall extend some results, which were previously known in the case of quasiconformal mappings, to the case of Dirichlet finite functions (cf. [21], [24]).

A function $u: R_{+}^{n} \rightarrow R$ is said to have a finite Dirichlet integral (or to be Dirichlet finite) if $u$ is $\mathrm{ACL}^{n}$ and if

$$
\int_{R_{+}^{n}}|\nabla u|^{n} d m<\infty
$$

In this paper all functions are required to be continuous. We shall consider the following weaker condition: $u$ is $\mathrm{ACL}^{n}$ and there are numbers $M, B \in(0, \infty)$ such that

$$
\int_{D(x, M)}|\nabla u|^{n} d m \leqq B
$$

for all $x \in R_{n}^{+}$, where $D(x, M)$ is the hyperbolic ball in $R_{+}^{n}$ with the centre $x$ and radius $M$. If (1.2) holds, $u$ is said to have a locally bounded Dirichlet integral.

In the preliminary Section 2 we prove, using the modulus method, that functions of $R_{+}^{n}$ which are monotone (in the sense of Lebesgue) and have a locally bounded Dirichlet integral are uniformly continuous with respect to the hyperbolic metric of $R_{+}^{n}$. In Section 3 we prove that a monotone function satisfying (1.1) and having a limit $\alpha$ at 0 through a set $E \subset R_{+}^{n}$, has in fact an angular limit $\alpha$ at 0 provided that the lower capacity density of $E$ at 0 is positive, cap dens $(E, 0)>0$. Similar results for some other classes of functions were given in [21] and [22]. An example is given to show that the condition cap $\overline{\operatorname{dens}}(E, 0)>0$ is not sufficient here. As an application of the results of Section 3 we can prove that a Dirichlet finite quasiregular mapping 
$f: R_{+}^{n} \rightarrow R^{n}, f=\left(f_{1}, \ldots, f_{n}\right)$, will have an angular limit $\left(\alpha_{1}, \ldots, \alpha_{n}\right)$ at 0 if each coordinate function $f_{j}$ has a limit $\alpha_{j}$ through a set $E_{j}$ with cap dens $\left(E_{j}, 0\right)>0$. A related result for bounded analytic functions was proved by F. W. Gehring and A. J. Lohwater [4]. In Section 4 we shall give an example illustrating the behaviour of a monotone function satisfying (1.2). The topic of Section 5 is the behaviour of a monotone function at an isolated singularity. In the final section, Section 6, we prove a variant of the Iversen-Tsuji theorem for monotone Dirichlet finite functions.

\section{Preliminary results}

We shall follow, as a rule, the notation and terminology of Väisälä’s book [18], which the reader is referred to for some definitions etc. Some notation will be introduced at first.

2.1. For $x \in R^{n}, n \geqq 2$, and $r>0$, let $B^{n}(x, r)=\left\{z \in R^{n}:|z-x|<r\right\}, S^{n-1}(x, r)=$ $\partial B^{n}(x, r), \quad B^{n}(r)=B^{n}(0, r), \quad S^{n-1}(r)=\partial B^{n}(r), \quad B^{n}=B^{n}(1), \quad$ and $\quad S^{n-1}=\partial B^{n}$. If $x \in R^{n}$ and $b>a>0$, then we write $R(x, b, a)=B^{n}(x, b) \backslash \bar{B}^{n}(x, a)$ and $R(b, a)=$ $R(0, b, a)$. The standard coordinate unit vectors are $e_{1}, \ldots, e_{n}$. If $A \subset R^{n}$, let $A_{+}=\left\{x=\left(x_{1}, \ldots, x_{n}\right) \in A: x_{n}>0\right\}$. The hyperbolic metric $\varrho$ in $R_{+}^{n}$ is defined by the element of length $d \varrho=|d x| / x_{n}$. If $x \in R_{+}^{n}$ and $M>0$, we write $D(x, M)=\left\{z \in R_{+}^{n}\right.$ : $\varrho(z, x)<M\}$. A well-known fact is that the hyperbolic balls are balls in the euclidean geometry as well, for instance

$$
D\left(t e_{n}, M\right)=B^{n}\left((t \cosh M) e_{n}, t \sinh M\right), \quad t>0 .
$$

For $x, y \in R_{+}^{n}$ the following formula holds [2, (3.3.4) p. 35]:

$$
\cosh \varrho(x, y)=1+\frac{|x-y|^{2}}{2 x_{n} y_{n}} .
$$

Sometimes we shall regard $B^{n}$ as a hyperbolic space as well and use the same symbols as in the case of $R_{+}^{n}$. The hyperbolic metric $\varrho$ is then defined by $d \varrho=2|d x| /\left(1-|x|^{2}\right)$. The counterpart of (2.2) for $B^{n}$ is

$$
D(x, M)=B^{n}(y, r)\left\{\begin{array}{l}
y=\frac{x\left(1-\tanh ^{2}(M / 2)\right)}{1-|x|^{2} \tanh ^{2}(M / 2)} \\
r=\frac{\left(1-|x|^{2}\right) \tanh (M / 2)}{1-|x|^{2} \tanh ^{2}(M / 2)} .
\end{array}\right.
$$

2.5. Monotone functions. Let $G \subset R^{n}$ be an open set. A continuous function $u: G \rightarrow R$ is said to be monotone (in the sense of Lebesque) if

$$
\max _{\bar{D}} u(x)=\max _{\partial D} u(x), \quad \min _{\bar{D}} u(x)=\min _{\partial D} u(x),
$$

whenever $D$ is a domain with compact closure $\bar{D} \subset G$. 
2.6. Remark. It follows from the above definition that if $t \in R$, then each component $A \neq \emptyset$ of the set $\{z \in G: u(z)>t\}$ is not relatively compact, i.e., $\bar{A} \cap(\partial G \cup$ $\{\infty\}) \neq \emptyset$. A similar statement holds if $>$ is replaced by $\geqq,<$, or $\leqq$. Hence monotone functions obey a sort of maximum principle.

2.7. The modulus of a curve family. Let $\Gamma$ be a family of curves in $R^{n}$. The modulus of $\Gamma$ is defined by

$$
M(\Gamma)=\inf _{\varrho \in F(\Gamma)} \int_{R^{n}} \varrho^{n} d m,
$$

where $F(\Gamma)$ is the family of all non-negative Borel functions $\varrho: R^{n} \rightarrow R^{1} \cup\{\infty\}$ with $\int_{\gamma} \varrho d s \geqq 1$ for all locally rectifiable $\gamma \in \Gamma$. For the properties of the modulus the reader is referred to Väisälä's book [18]. If $E, F, G \subset R^{n}$, then $\Delta(E, F ; G)$ is the family of all curves $\gamma \subset G$ joining $E$ to $F$ in the following sense: $\bar{\gamma} \cap E \neq \emptyset \neq \bar{\gamma} \cap F$.

2.8. Condenser and its capacity. A pair $(A, C)$ is said to be a condenser if $A \subset R^{n}$ is open and $C \subset A$ is compact. The capacity of $E=(A, C)$ is defined by

$$
\operatorname{cap} E=\operatorname{cap}(A, C)=\inf _{u} \int_{R^{n}}|\nabla u|^{n} d m,
$$

where $u$ runs through the set of all $\mathrm{ACL}^{n}$ functions with compact support in $A$ and with $u(x) \geqq 1$ for $x \in C$. An alternative definition is

$$
\operatorname{cap}(A, C)=M\left(\Delta\left(C, \partial A ; R^{n}\right)\right)=M(\Delta(C, \partial A ; A)) .
$$

Ziemer [26] proved that (2.9) and (2.10) agree for bounded $A$, from which the same conclusion for unbounded $A$ follows easily. A compact set $E$ in $R^{n}$ is said to be of capacity zero if $\operatorname{cap}\left(B^{n}(t), E\right)=0$ for some $t>0$ such that $E \subset B^{n}(t)$.

The following lower bound for the Dirichlet integral of an $\mathrm{ACL}^{n}$ function will be applied several times in what follows. In fact, it is the easy part in the proof of (2.10). The proof is standard (cf. [1, p. 65], [9, p. 577], [26, Lemma 3.1]).

2.11. Lemma. Let $u: G \rightarrow R$ be an $\mathrm{ACL}^{n}$ function, $-\infty<a<b<\infty$, and let $A, B \subset G$ be such that $u(x) \leqq a$ for $x \in A$ and $u(x) \geqq b$ for $x \in B$. Then

$$
M(\Delta(A, B ; G)) \leqq(b-a)^{-n} \int_{G}|\nabla u|^{n} d m .
$$

2.12. Remark. In classical complex analysis one often uses a different capacity. The connection between the classical capacity and the above one has been studied in $[1$, p. 70] and in [13].

We next give a proof of the fact that a monotone $\mathrm{ACL}^{n}$ function with a locally bounded Dirichlet integral is uniformly continuous with respect to the hyperbolic metric. For the sake of technical reasons we shall consider functions defined in $B^{n}$, but with small modifications one can prove a similar result for functions defined in $R_{+}^{n}$. 
2.13. Lemma. Let $u: B^{n} \rightarrow R$ be a monotone $\mathrm{ACL}^{n}$ function satisfying (1.2). Then

$$
|u(x)-u(y)|^{n} \leqq C\left(\log \frac{1}{r}\right)^{-1}(1-r)^{1-n},
$$

where $r=\tanh (\varrho(x, y) / 4)$ and $C$ is a positive constant depending only on $n, M$, and $B$ in (1.2). In particular, $u:\left(B^{n}, \varrho\right) \rightarrow(R,||)$ is uniformly continuous.

Proof. Clearly we may assume $u(x)<u(y)$. Since the right side depends only on $x$ and $y$ through the Möbius invariant quantity $\varrho(x, y)$, we may assume $x=r e_{1}=$ $-y, \quad r=\tanh (\varrho(x, y) / 4)($ cf. $(2.4))$. Let

$$
\begin{aligned}
& E=\left\{z \in B^{n}: u(z) \leqq u(x)\right\}, \\
& F=\left\{z \in B^{n}: u(z) \geqq u(y)\right\}, \\
& \Gamma_{\boldsymbol{r}}=\Delta\left(E, F ; B^{n}(\sqrt{r})\right) .
\end{aligned}
$$

Then by Remark 2.6 and [18, 10.12]

Lemma 2.11 yields

$$
M\left(\Gamma_{r}\right) \geqq c_{n} \log \frac{1}{\sqrt{r}} .
$$

$$
M\left(\Gamma_{r}\right) \leqq|u(x)-u(y)|^{-n} \int_{B^{n}(\sqrt{r})}|\nabla u|^{n} d m .
$$

In view of (1.2) the integral can be estimated from above in terms of $B$ and the following number

$$
k=\inf \left\{p: \overline{B^{n}(\sqrt{r})} \subset \bigcup_{j=1}^{p} D\left(x_{j}, M\right)\right\} .
$$

An upper bound for $k$ can be found by a method involving estimation of the hyperbolic volume of $\cup\{D(x, M):|x| \leqq \sqrt{r}\}$. (For details see [25, Section 9]). This method yields the estimate

$$
\int_{B^{n}(\sqrt{r})}|\nabla u|^{n} d m \leqq d(1-\sqrt{r})^{1-n},
$$

where $d$ is a positive number depending only on $n, M$, and $B$. The desired estimate with $C=2^{n} d / c_{n}$ follows from this and the preceding estimates.

The uniform continuity in 2.13 can also be proved with the help of an oscillation inequality of Gehring [3, p. 355]. (See also Lelong-Ferrand [7, p. 7]). For $n$-dimensional version of the oscillation inequality see Mostow [12]. The oscillation inequality is applied in the proof of $[18,10.12]$, which was exploited above.

2.14. Remark. For large values of $\varrho(x, y)$ the above upper bound is not sharp. Indeed, one can replace the factor $(\log (1 / r))^{-1}(1-r)^{1-n}$ in 2.13 by $(1+\log ((1+r) /(1-r))) \cdot$ const., which yields a better estimate for large values of 
$Q(x, y)$. Such an estimate can be deduced from the above version of Lemma 2.13 , in particular, from the fact that $u$ is uniformly continuous.

A continuous function $v: R_{+}^{n} \rightarrow R_{+} \cup\{0\}, R_{+}=\{x \in R: x>0\}$, is said to satisfy a Harnack inequality if there exist numbers $\lambda \in(0,1)$ and $C_{\lambda} \geqq 1$ such that (cf. [23])

$$
\max _{\bar{B}^{n}(x, \lambda r)} v(z) \leqq C_{\lambda} \min _{\bar{B}^{n}(x, \lambda r)} v(z)
$$

whenever $B^{n}(x, r) \subset R_{+}^{n}$. The next result should be compared with [5, p. 200].

2.16. Corollary. If $u: R_{+}^{n} \rightarrow R$ is a monotone function satisfying (1.2), then $e^{u}$ satisfies $(2.15)$ for every $\lambda \in(0,1)$ with

$$
\log C_{\lambda}=C^{1 / n}\left(\log \frac{1}{\lambda}\right)^{-1 / n}\left(\frac{1+\lambda}{1-\lambda}\right)^{(n-1) /(2 n)},
$$

where $C$ is the number in 2.13 .

Proof. Fix $\lambda \in(0,1)$. Choose $B^{n}(x, r) \subset R_{+}^{n}$. Then $r \leqq x_{n}$, where $x=\left(x_{1}, \ldots\right.$ $\left.\ldots, x_{n}\right)$, and $\varrho\left(\bar{B}^{n}(x, \lambda r)\right) \leqq \log ((1+\lambda) /(1-\lambda))$ by (2.2) or (2.3). For $z, y \in \bar{B}^{n}(x, \lambda r)$ we get by 2.13 and by some elementary inequalities that

$$
|u(z)-u(y)| \leqq C^{1 / n}\left(\log \frac{1}{\lambda}\right)^{-1 / n}\left(\frac{1+\lambda}{1-\lambda}\right)^{(n-1) /(2 n)},
$$

from which (2.15) for $e^{u}$ follows.

We shall now give examples of functions satisfying (1.2) in $B^{n}$.

2.17. The function $u_{F}$. Let $F$ be a relatively closed subset of $B^{n}$. For $x \in B^{n}$ set (cf. $[23,3.6])$

$$
u_{F}(x)=\exp (-\varrho(x, F)) .
$$

As shown in [23], the function $u_{F}$ has some extremal properties for appropriate choices of $F$. For $x, y \in B^{n}$ and $\varrho(x, F) \leqq \varrho(y, F)$ we get

$$
\begin{gathered}
\left|u_{F}(x)-u_{F}(y)\right| \leqq\left|e^{-\varrho(x, F)}-e^{-\varrho(y, F)}\right|=e^{-\varrho(x, F)}\left|1-e^{-a}\right| \\
\leqq e^{-\varrho(x, F)} a \leqq e^{-\varrho(x, F)} \varrho(x, y) \leqq \varrho(x, y),
\end{gathered}
$$

where $a=\varrho(y, F)-\varrho(x, F)$. Similarly $\left|u_{F}(x)-u_{F}(y)\right| \leqq \varrho(x, y)$ for $\varrho(x, F) \geqq$ $Q(y, F)$ as well. Next we apply $[22,2.11]$ to get

$$
\varrho(x, y) \leqq \log \left(1+\frac{|x-y|}{d(x)-|x-y|}\right) \leqq \frac{|x-y|}{d(x)-|x-y|},
$$

for $y \in B^{n}(x, d(x))$ where $d(x)=d\left(x, \partial B^{n}\right)$. Therefore

$$
\limsup _{y \rightarrow x} \frac{\left|u_{F}(x)-u_{F}(y)\right|}{|x-y|} \leqq \limsup _{y \rightarrow x} \frac{1}{d(x)-|x-y|}=\frac{1}{d(x)} .
$$


It follows that $u_{F}$ is locally Lipschitz continuous and hence $\mathrm{ACL}^{n}$. Fix $M>0$. Then we get by (2.4) that

$$
\int_{D(x, M)}\left|\nabla u_{F}(x)\right|^{n} d m(x) \leqq \int_{D(x, M)} d(x)^{-n} d m(x) \leqq c_{1}(n, M) .
$$

In conclusion, $u_{F}$ satisfies (1.2). Note, however, that $u_{F}$ is usually not monotone.

2.18. Remark. It follows from [5, p. 23, formula (2.32)] and (2.4) that all bounded harmonic functions satisfy (1.2).

\section{Behaviour at an individual boundary point}

For $\varphi \in(0, \pi / 2)$ let $C(\varphi)=\left\{y \in R_{+}^{n}:\left(y \mid e_{n}\right)>|y| \cos \varphi\right\}$, where $(z \mid u)$ is the inner product $\sum z_{i} u_{i}$. A function $u: R_{+}^{n} \rightarrow R$ is said to have an angular limit $\alpha$ at 0 if, for each $\varphi \in(0, \pi / 2), \lim _{x \rightarrow 0, x \in C(\varphi)} u(x)=\alpha$. A function $u$ is said to have an asymptotic value $\alpha$ at 0 if there exists a continuous curve $\gamma:[0,1) \rightarrow R_{+}^{n}$ with $u(\gamma(t)) \rightarrow \alpha$ and $\gamma(t) \rightarrow 0$ as $t \rightarrow 1$.

3.1. Lemma. Let $u: R_{+}^{n} \rightarrow R$ be a monotone $\mathrm{ACL}^{n}$ function satisfying (1.2) and let $E \subset R_{+}^{n}$ be a measurable set such that

$$
\lim _{r \rightarrow 0} m\left(\left(R_{+}^{n} \backslash E\right) \cap B^{n}(r)\right) r^{-n}=0 .
$$

If $u(x) \rightarrow \alpha$ as $x \rightarrow 0$ and $x \in E$, then $u$ has an angular limit $\alpha$ at 0 .

Proof. The proof follows from 2.13 and [23, 6.13].

3.2. Remarks. (1) It is not difficult to show that the assumption in 3.1 is equivalent to the condition that $u$ has an approximate limit $\alpha$ at $0[23,6.3]$. For the definition of an approximate limit see [23,6.1]. For a related result see J. Lelong-Ferrand $[7$, p. 16] and $[21,5.9]$.

(2) The monotone $\mathrm{ACL}^{2}$ function $u: R_{+}^{2} \rightarrow R, u(x, y)=\overline{\operatorname{arc}} \tan (y / x)$, satisfies (1.2) (but not (1.1)) and has infinitely many distinct asymptotic values at 0 but no angular limit at 0 . Hence an approximate limit in the hypotheses of 3.1 cannot be replaced by an asymptotic value.

For $E \subset R^{n}, x \in R^{n}$, and $t>r>0$ set

$$
\begin{gathered}
M_{t}(E, r, x)=M\left(\Delta\left(S^{n-1}(x, t), E \cap \bar{B}^{n}(x, r) ; R^{n}\right)\right), \\
M(E, r, x)=M_{2 r}(E, r, x) .
\end{gathered}
$$

The lower and upper capacity densities of $E$ at $x$ are defined by

$$
\begin{aligned}
& \text { cap dens }(E, x)=\liminf _{r \rightarrow 0} M(E, r, x), \\
& \text { cap } \overline{\operatorname{dens}}(E, x)=\limsup _{r \rightarrow 0} M(E, r, x) .
\end{aligned}
$$


3.3. Lemma. Let $u:\left(R_{+}^{n}, \varrho\right) \rightarrow(R,||)$ be uniformly continuous, let $b_{k} \in R_{+}^{n}$, $b_{k} \rightarrow 0$, and let $u\left(b_{k}\right) \rightarrow \beta \neq \infty$. For every $\varepsilon>0$ there exists $M \in(0, \infty)$ and $p \geqq 1$ such that

$$
|u(x)-\beta|<\varepsilon, \quad x \in E_{M}=\bigcup_{k \geqq p} D\left(b_{k}, M\right) .
$$

Moreover, if there exists $\varphi \in(0, \pi / 2)$ such that $b_{k} \in C(\varphi)$ for all $k$, then there exists a positive number $c$ depending only on $n, \varphi$, and $M$ such that

$$
\operatorname{cap} \overline{\operatorname{dens}}\left(E_{M}, 0\right) \geqq c>0 .
$$

Proof. The first part follows from the definition of uniform continuity. For the proof of the second part we assume $b_{k} \in C(\varphi), k=1,2, \ldots$ It follows from (2.2) that $F_{M} \subset E_{M}$,

$$
F_{M}=\bigcup_{k \geqq p} B^{n}\left(b_{k}, b_{k n}\left(1-e^{-M}\right)\right),
$$

where $b_{k n} \geqq\left|b_{k}\right| \cos \varphi$ is the $n$-th coordinate of $b_{k}$. Hence the proof follows from [18; 10.12]. For more details see [21, 2.5 (2)].

3.4. Theorem. Let $u:\left(R_{+}^{n}, \varrho\right) \rightarrow(R,||)$ be a uniformly continuous and Dirichlet finite function and let $E \subset R_{+}^{n}$ be a set with cap dens $(E, 0)>0$. If $u(x) \rightarrow \alpha$ as $x \rightarrow 0$, $x \in E$, then $u$ has an angular limit $\alpha$ at 0 .

Proof. Fix $\varphi \in(0, \pi / 2)$. Suppose, on the contrary, that there exists a sequence $\left(b_{k}\right)$ in $C(\varphi)$ with $b_{k} \rightarrow 0$ and $u\left(b_{k}\right) \rightarrow \beta \neq \alpha$. We shall assume that $-\infty<\alpha<\beta<\infty$; in other cases the proof is similar. Let $B_{k}$ be the $b_{k}$-component of the set $B=\left\{z \in R_{+}^{n}: u(z)>(\alpha+2 \beta) / 3\right\}$ and let $A=\left\{z \in R_{+}^{n}: u(z)<(2 \alpha+\beta) / 3\right\}$. Since $u$ is uniformly continuous, there exist by Lemma 3.3 numbers $M>0$ and $p \in \mathbf{N}$ such that $D\left(b_{k}, M\right) \subset B_{k}$ for $k \geqq p$ and

$$
\operatorname{cap} \overline{\operatorname{dens}}(B, 0) \geqq c>0 .
$$

Since cap dens $(E, 0)>0$, it follows from [21, 4.3] and [20, 3.8] that

$$
M\left(\Delta\left(A, B ; R_{+}^{n}\right)\right) \geqq M\left(\Delta\left(A, B ; R^{n}\right)\right) / 2=\infty .
$$

A contradiction follows from (1.1) and 2.11.

3.5. Remark. The condition cap dens $(E, 0)>0$ is satisfied for instance if $E$ is a curve terminating at 0 . This fact follows from $[18,10.12]$; for details and other sufficient conditions for cap dens $(E, 0)>0$ see [21]. On the other hand, there are compact sets $E$ of zero Hausdorff dimension with cap dens $(E, 0)>0$ (cf. [21, 2.5 (3)]).

We shall now construct an example showing that the condition cap $\overline{\operatorname{dens}}(E, 0)>0$ would not suffice in 3.4 . 
3.6. Example. There exists a monotone $\mathrm{ACL}^{n}$ function $u: R_{+}^{n} \rightarrow R$ with a finite Dirichlet integral such that for a sequence $r_{k} \rightarrow 0$ with $0<r_{k+1}<r_{k}$

$$
\begin{gathered}
u \mid \bigcup_{k=1}^{\infty} S_{+}^{n-1}\left(r_{2 k-1}\right)=1, \\
u \mid \bigcup_{k=1}^{\infty} S_{+}^{n-1}\left(r_{2 k}\right)=0 .
\end{gathered}
$$

Let $r_{1}=1$. Select $r_{k+1} \in\left(0, r_{k} / 2\right), k=1,2, \ldots$, such that

$$
\left(\log \frac{r_{k}}{r_{k+1}}\right)^{1-n}<2^{-k} \text {. }
$$

Define $u: R_{+}^{n} \rightarrow R$ by $u(x)=1$ for $x \in R_{+}^{n} \backslash B^{n}$ and

$$
\begin{array}{ll}
u(x)=\frac{\log |x|-\log r_{2 k}}{\log \left(r_{2 k-1} / r_{2 k}\right)} ; & r_{2 k-1}>|x| \geqq r_{2 k}, \quad x \in R_{+}^{n}, \\
u(x)=\frac{\log r_{2 k}-\log |x|}{\log \left(r_{2 k} / r_{2 k+1}\right)} ; \quad r_{2 k}>|x| \geqq r_{2 k+1}, & x \in R_{+}^{n},
\end{array}
$$

for $k=1,2, \ldots$ It follows from (3.7) that (1.1) holds (cf. [18, 7.5]). Clearly $u$ is monotone and satisfies (a) and (b). In addition, cap $\overline{\operatorname{dens}}(B, 0)>0, B=\bigcup_{k=1}^{\infty} S_{+}^{n-1}\left(r_{2 k}\right)$ and $u(z) \rightarrow 0$, as $z \rightarrow 0, z \in B$, and $u$ fails to have an asymptotic value and hence an angular limit at 0 . Therefore the condition cap dens $(E, 0)>0$ in Theorem 3.4 cannot be replaced by cap $\overline{\operatorname{dens}}(E, 0)>0$.

3.8. Theorem. Let $u: R_{+}^{n} \rightarrow R$ be a monotone and Dirichlet finite function, let $\left(b_{k}\right) \subset R_{+}^{n}$ be a sequence with $\varrho\left(b_{k}, b_{h}\right) \geqq 4 M$ for $k \neq h$, and let $a_{k} \in D\left(b_{k}, M\right)$. For each $\varepsilon>0$ let $P_{\varepsilon}=\left\{k \in N:\left|u\left(a_{k}\right)-u\left(b_{k}\right)\right| \geqq \varepsilon\right\}$. Then

$$
\operatorname{card} P_{\varepsilon} \leqq A \varepsilon^{-n} \int_{R_{+}^{n}}|\nabla u|^{n} d m,
$$

where $A$ is a positive number depending only on $n$ and $M$.

Proof. Fix $\varepsilon>0$. Let

$$
\begin{gathered}
A_{k}=\left\{z \in R_{+}^{n}:\left|u(z)-u\left(a_{k}\right)\right|<\varepsilon / 3\right\}, \\
B_{k}=\left\{z \in R_{+}^{n}:\left|u(z)-u\left(b_{k}\right)\right|<\varepsilon / 3\right\}, \\
\Gamma_{k}=\Delta\left(A_{k}, B_{k} ; D\left(b_{k}, 2 M\right)\right), \quad k \in P_{\varepsilon} .
\end{gathered}
$$

From 2.6 it follows that the $a_{k}$-component of $A_{k}$ meets $\partial D\left(b_{k}, 2 M\right)$, and that so does the $b_{k}$-component of $B_{k}$, when $k \in P_{\varepsilon}$. It follows from the conformal invariance of the modulus $[18,8.1]$, from $\varrho\left(a_{k}, b_{k}\right)<M$, and from (2.4) that

$$
M\left(\Gamma_{k}\right) \geqq c_{n} \log \frac{\tanh M}{\tanh (M / 2)} \geqq c_{n}(\log 2) e^{-M}
$$


holds for $k \in P_{\varepsilon}$. Let $A=\bigcup_{k \in P_{\varepsilon}} A_{k}, B=\bigcup_{k \in P_{\varepsilon}} B_{k}$ and $\Gamma=\Delta\left(A, B ; R_{+}^{n}\right)$. Since $\varrho\left(b_{k}, b_{h}\right) \geqq 4 M$ for $k \neq h$, it follows that $\left\{\Gamma_{k}: k \in P_{\varepsilon}\right\}$ are separate subfamilies of $\Gamma$. Hence we get by $[18,6.7]$

$$
\sum_{K \in P_{\varepsilon}} M\left(\Gamma_{k}\right) \leqq M(\Gamma) \leqq\left(\frac{3}{\varepsilon}\right)^{n} \int_{R_{+}^{n}}|\nabla u|^{n} d m .
$$

The desired upper bound for card $P_{\varepsilon}$ follows from this and the preceding inequality with $A=\left(3^{n} e^{M}\right) /\left(c_{n} \log 2\right)$.

3.9. Remark. A similar result holds for a uniformly continuous Dirichlet finite function $u:\left(R_{+}^{n}, \varrho\right) \rightarrow(R,||)$ as well, but with a more complicated dependence on the Dirichlet integral and the modulus of continuity of the function.

3.10. Theorem. Let $u:\left(R_{+}^{n}, \varrho\right) \rightarrow(R,||)$ be a uniformly continuous and Dirichlet finite function, let $\left(b_{k}\right) \subset R_{+}^{n}, b_{k} \rightarrow 0, u\left(b_{k}\right) \rightarrow \beta$ and let $M \in(0, \infty)$. Then $u(x) \rightarrow \beta$ as $x \rightarrow 0, x \in \cup D\left(b_{k}, M\right)$.

Proof. In the case of monotone functions the proof follows from 3.8. The general case follows from 3.9 .

The next theorem has its roots in [24], where a similar result was proved for quasiconformal mappings. We shall omit the proof since it parallels the proofs of Theorems 3.8 and that of $[24,4.9]$. For the statement of the theorem the following condition is needed. Let $\left(a_{k}\right)$ and $\left(b_{k}\right)$ be sequences in $R_{+}^{n}$ tending to 0 and let $J_{k}=J\left[a_{k}, b_{k}\right]$ be the closed geodesic segment in the hyperbolic geometry joining $a_{k}$ with $b_{k}$. Thus $J_{k}$ is the arc between $a_{k}$ and $b_{k}$ on a circle through $a_{k}$ and $b_{k}$, which is orthogonal to $\partial R_{+}^{n}$. Suppose that there exists a positive number $M$ such that

$$
\varrho\left(J_{k}, J_{h}\right) \geqq M>0 \text { for } k \neq h .
$$

As in [24, 4.9], condition (3.11) is needed to guarantee that some curve families are separate.

3.12. Theorem. Let $\left(a_{k}\right)$ and $\left(b_{k}\right)$ be sequences in $R_{+}^{n}$ tending to 0 and satisfying (3.11), let $u:\left(R_{+}^{n}, \varrho\right) \rightarrow(R,||)$ be uniformly continuous and Dirichlet finite, and let $u\left(a_{k}\right) \rightarrow \alpha, u\left(b_{k}\right) \rightarrow \beta$. If $\sum \varrho\left(a_{k}, b_{k}\right)^{1-n}=\infty$, then $\alpha=\beta$.

Proof. The proof is similar to the proof of 3.8 and $[24,4.9]$. The details are left to the reader.

3.13. Remarks. (1) Theorem 3.4 was proved by V. M. Mikljukov [10] in the case when the set $E$ is a curve and the mapping is vector-valued and of class BL. One variant of Theorem 3.10 for these mappings was given by G. D. Suvorov [17, p. 122].

(2) It should be observed that Theorem 3.12 follows from Theorem 3.10 in the case $\lim \inf \varrho\left(a_{k}, b_{k}\right)<\infty$. 
(3) We shall next show that condition (3.11) cannot be removed from the hypotheses of Theorem 3.12 even in the case of continuous mappings (and, a fortiori, in the case of uniformly continuous Dirichlet finite functions). Let $u: R_{+}^{n} \rightarrow R$ be a continuous function such that $u\left(\tilde{a}_{k}\right) \rightarrow 0, u\left(\tilde{b}_{k}\right) \rightarrow 1$, where $\tilde{a}_{k}, \tilde{b}_{k} \rightarrow 0$. We shall construct two new sequences $\left(a_{k}\right)$ and $\left(b_{k}\right)$ with $\left\{a_{j}\right\}=\left\{\tilde{a}_{k}\right\}$ and $\left\{b_{j}\right\}=\left\{\tilde{b}_{k}\right\}$ for which (3.11) fails to hold and for which $\sum \varrho\left(a_{k}, b_{k}\right)^{1-n}=\infty$. To this end, let $p_{1}=1, p_{j+1}>p_{j}$ be an increasing sequence of integers such that

for all $k=1,2, \ldots$ Set

$$
\left(p_{k+1}-p_{k}\right) \varrho\left(\tilde{a}_{k}, \tilde{b}_{k}\right)^{1-n}>1 / k
$$

$$
a_{j}=\tilde{a}_{i} \text { and } b_{j}=\tilde{b}_{i} \quad \text { if } \quad p_{i} \leqq j<p_{i+1} .
$$

Then $a_{k}, b_{k} \rightarrow 0$ and $\sum \varrho\left(a_{k}, b_{k}\right)^{1-n}=\infty$ hold while $u\left(a_{k}\right) \rightarrow 0, u\left(b_{k}\right) \rightarrow 1$.

We show that Theorem 3.12 is sharp in a sense.

3.14. Theorem. Let $\left(b_{k}\right)$ be a sequence in $R_{+}^{n}$ with $\left|b_{k+1}\right|<\left|b_{k}\right|, b_{k} \rightarrow 0$, let $a_{k}=\left|b_{k}\right| e_{n}$, and suppose that (3.11) holds. If $\sum \varrho\left(a_{k}, b_{k}\right)^{1-n}<\infty$, then there exists a monotone Dirichlet finite function $u: R_{+}^{n} \rightarrow R$ having an angular limit 0 at 0 and satisfying $u\left(b_{k}\right) \rightarrow 1$.

Proof. Since $a_{k}=\left|b_{k}\right| e_{n}$, we obtain by (3.11), (2.2), and (2.3) that

$$
\varrho\left(J_{k}, J_{k+1}\right)=\log \frac{\left|b_{k}\right|}{\left|b_{k+1}\right|} \geqq M>0 .
$$

It follows that the annuli $R\left(\left|b_{k}\right| \lambda,\left|b_{k}\right| / \lambda\right)$ are disjoint when $\lambda=e^{M / 2}$. Let $w_{k} \in \partial R_{+}^{n}$ be a unit vector such that $b_{k}-b_{k n} e_{n}=c w_{k}$, where $b_{k n}$ is the $n$-th coordinate of $b_{k}$ and $c$ is a positive number such that $\left|b_{k}\right|^{2}=c^{2}+b_{k n}^{2}$. The balls $B_{k}=B^{n}\left(\left|b_{k}\right| w_{k}, r_{k}\right), r_{k}=$ $\left|b_{k}\right|(1-1 / \lambda)$ are then disjoint. Let $t_{k}=\left|b_{k}-\right| b_{k}\left|w_{k}\right|$. It follows from (2.3) that

$$
t_{k}<2\left|b_{k}\right| \exp \left(-\varrho\left(a_{k}, b_{k}\right)\right)
$$

(for more details see [23, (2.4)]). Since $\sum \varrho\left(a_{k}, b_{k}\right)^{1-n}<\infty$, it follows that $\varrho\left(a_{k}, b_{k}\right) \rightarrow \infty$ as $k \rightarrow \infty$. By relabelling and passing to a subsequence if necessary we may hence assume, in view of the above estimate for $t_{k}$, that $t_{k}<r_{k}$ for all $k$. Choose now a monotone $\mathrm{ACL}^{n}$ function $u_{k}$ such that (cf. 3.6)

$$
\begin{gathered}
u_{k}\left|R_{+}^{n} \backslash B_{k}=0, \quad u_{k}\right| R_{+}^{n} \cap \bar{B}^{n}\left(\left|b_{k}\right| w_{k}, t_{k}\right)=1, \\
d_{k}=\int_{R_{+}^{n}}\left|\nabla u_{k}\right|^{n} d m=\frac{\omega_{n-1}}{2}\left(\log \frac{r_{k}}{t_{k}}\right)^{1-n} .
\end{gathered}
$$

There exist numbers $k_{0}$ and $c(n, M)$ such that for $k \geqq k_{0}$

$$
d_{k} \leqq c(n, M) \varrho\left(a_{k}, b_{k}\right)^{1-n} .
$$

Set $u=\sum_{k \geqq k_{0}} u_{k}$. Then $u$ is monotone, $\mathrm{ACL}^{n}, u\left(b_{k}\right) \rightarrow 1, u\left(t e_{n}\right)=0, t>0$ and $u$ has a finite Dirichlet integral, as desired. 
We shall next give some applications of the preceding results to the theory of quasiregular mappings. A continuous $\mathrm{ACL}^{n}$ mapping $f: R_{+}^{n} \rightarrow R^{n}$ is called quasiregular (qr) if there exists a constant $K \in[1, \infty)$ such that

$$
\sup _{|h|=1}\left|f^{\prime}(x) h\right|^{n} \leqq K J_{f}(x)
$$

a.e. in $R_{+}^{n}$, where $J_{f}$ is the Jacobian determinant of $f$. A sense-preserving homeomorphism is quasiregular if and only if it is quasiconformal (qc). For the basic parts of the theory of qc and qr mappings the reader is referred to [15], [18], [19]. For the following result see Rešetnjak's book [15, p. 118].

3.15. Lemma. The coordinate functions $f_{1}, \ldots, f_{n}$ of a qr mapping $f: R_{+}^{n} \rightarrow R^{n}$, $f=\left(f_{1}, \ldots, f_{n}\right)$, are monotone.

3.16. Theorem. Let $f: R_{+}^{n} \rightarrow R^{n}$ be a qr mapping with

$$
\int_{R_{+}^{n}}\left|\nabla f_{j}\right|^{n} d m<\infty, \quad j=1, \ldots, n \text {. }
$$

If $f_{j}(x) \rightarrow \alpha_{j}$ as $x \rightarrow 0, x \in E_{j}$ and cap dens $\left(E_{j}, 0\right)>0$, then $f_{j}$ has an angular limit $\alpha_{j}$ at $0, j=1,2, \ldots, n$.

Proof. The proof follows from 3.15, 2.13, and 3.4.

3.17. Remarks. For bounded analytic functions a result similar to 3.16 holds without a condition about finite Dirichlet integral (Gehring-Lohwater [4]). In the case of bounded qr mappings $f: R_{+}^{n} \rightarrow R^{n}$ such a condition is, however, necessary if $n \geqq 3$. This fact follows from an example due to Rickman [16].

\section{On the behaviour at a typical boundary point}

In this section we shall study the behaviour of a Dirichlet finite function at a "typical" boundary point. We shall employ the following result of Rešetnjak [14].

4.1. Lemma. Let $u: R_{+}^{n} \rightarrow R$ be an $\mathrm{ACL}^{n}$ function with a finite Dirichlet integral. Then there exists a set $E \subset \partial R_{+}^{n}$ such that every compact set $F$ in $E$ is of zero $n$-capacity and such that $u$ has an essential value at every point of $\partial R_{+}^{n} \backslash E$, i.e., for every $x \in \partial R_{+}^{n} \backslash E$ there exists a number $\alpha$ with

$$
\lim _{r \rightarrow 0} r^{-n} \int_{B_{+}^{n}(x, r)}|f(y)-\alpha| d m=0 .
$$

4.2. Theorem. Let $u: R_{+}^{n} \rightarrow R$ be a monotone $\mathrm{ACL}^{n}$ function with a finite Dirichlet integral. Then $u$ has an angular limit at every point of $\partial R_{+}^{n} \backslash E$, where $E$ is as in 4.1 . 
Proof. Since $u$ has an essential value at the points of $\partial R_{++}^{n} \backslash E$, it has an approximate limit as well by [23, 6.7 (1)]. By 3.1 and 3.2 (1) it has an angular limit, too.

We next show that 4.2 fails to hold for monotone functions satisfying (1.2) but $\operatorname{not}(1.1)$.

4.3. Example. There exists a bounded monotone $\mathrm{ACL}^{2}$ function $u: R_{+}^{2} \rightarrow R$ satisfying condition (1.2), having an asymptotic value at each point of a dense subset of $\partial R_{+}^{n}$, but having no angular limits.

Divide the square $Q=[0,1] \times(0,1] \subset R_{+}^{2}$ into four equal squares by joining the midpoints of opposite sides with (euclidean) segments. Repeat the division in those resulting squares which have one side on the $x$-axis. By continuing this process we get a division of $Q$ into closed squares $Q_{i}^{j}: i=1,2, \ldots, j=1, \ldots, 2^{i}$ of constant hyperbolic size, where $Q_{i}^{j}$ has euclidean side-length $2^{-i}$. Join the center of $Q_{i}^{j}$ by (euclidean) segments to the centres of those two adjacent squares in $\left\{Q_{i+1}^{j}: j=1,2, \ldots, 2^{i+1}\right\}$ each of which has a side lying on a side of $Q_{i}^{j}$, for each $i$ and $j$. As a result we get two distinct "treelike" infinite polygonal curves approaching the $x$-axis. The union of these curves will be denoted by $T$.

Define $u(x)=0$ if $x$ is located on a side $A$ of a square $Q_{i}^{j}$ and $A \cap T=\emptyset$ and $u(y)=1$ if $y \in T$. In (int $Q$ ) \T define $u$ in such a way that $u: Q \rightarrow R \cup[0,1]$ will be monotone, have all partial derivatives, continuous in $U\left(\right.$ int $\left.Q_{k}^{j}\right) \backslash T$ and

$$
|\nabla u(z)| \leqq 2^{k+3} \quad \text { for } \quad z \in\left(\text { int } Q_{k}^{j}\right) \backslash T,
$$

$j=1,2, \ldots, 2^{k}$. Extend the domain of definition of $u$ to $R_{+}^{2}$ as follows. If $\operatorname{Im} z>1$, set $u(z)=0$. If $p \in Z$ and $z \in Q+\{(p, 0)\}$, then $z-(p, 0) \in Q$; set $u(z)=u(z-(p, 0))$. Then $u$ is defined in $R_{+}^{2}$, has an asymptotic value 1 at the points of $\bar{T} \cap \partial R_{+}^{2} \backslash\{\infty\}$ through the set $T$ and is monotone, and it follows from (4.4) that (1.2) holds. Moreover, it is clear that $u$ has no angular limits.

\section{On isolated singularities and Phragmén-Lindelöf-type behaviour}

A function with a finite Dirichlet integral need not have a limit at an isolated singularity. To see this fact we may consider the function in Example 3.5 and extend it by reflection in $\partial R_{+}^{n}$ to a map $v: R^{n} \backslash\{0\} \rightarrow R$ with a finite Dirichlet integral and with no limit at 0 . This function is not, however, monotone although $v \mid R_{+}^{n}$ indeed is monotone.

5.1. Theorem. Let $u: R^{n} \backslash\{0\} \rightarrow R$ be a monotone $\mathrm{ACL}^{n}$ function. If $u$ has no limit at 0 , then

$$
\liminf _{t \rightarrow 0} \int_{R(1, t)}|\nabla u|^{n} d m / \log \frac{1}{t}>0
$$


Proof. Suppose that there are sequences $\left\{a_{k}\right\},\left\{b_{k}\right\}$ in $B^{n} \backslash\{0\}$ with $a_{k}, b_{k} \rightarrow 0$ and $u\left(a_{k}\right) \rightarrow \alpha, u\left(b_{k}\right) \rightarrow \beta \neq \alpha$. We may assume $-\infty<\alpha<\beta<\infty$. Let $A_{k}$ be the $a_{k}$ component of the set

$$
A=\left\{z \in R^{n} \backslash\{0\}: u(z) \leqq(2 \alpha+\beta) / 3\right\},
$$

and $B_{k}$ the $b_{k}$-component of the set

$$
B=\left\{z \in R^{n} \backslash\{0\}: u(z) \geqq(2 \beta+\alpha) / 3\right\} .
$$

By 2.6, $\bar{A}_{k} \cap\{0, \infty\} \neq \emptyset \neq \bar{B}_{k} \cap\{0, \infty\}$ for all large $k$. There is a sequence $\left(j_{k}\right)$ such that either $0 \in \bar{A}_{j_{k}}$ for all $j_{k}$ or $\infty \in \bar{A}_{j_{k}}$ for all $j_{k}$. Consider the first case, the proof being similar in the second case. For $t \in(0,1)$ set

$$
\Gamma_{t}=\Delta\left(B, A_{j_{1}} ; R(1, t)\right) .
$$

Suppose that $0 \in \bar{B}_{k}$ for some $k$ such that $\left|b_{k}\right|<\left|a_{j_{1}}\right|$. Then we get by $[18,10.12]$

$$
M\left(\Gamma_{t}\right) \geqq c_{n} \log \frac{\left|b_{k}\right|}{t}=c_{n} \log \left|b_{k}\right|+c_{n} \log \frac{1}{t} ; t<\left|b_{k}\right| .
$$

Otherwise $\infty \in \bar{B}_{k}$ for all $k$ such that $\left|b_{k}\right|<\left|a_{j_{1}}\right|$ and thus $S^{n-1}(r) \cap B \neq \emptyset$ for all $r \in\left(0,\left|a_{j_{1}}\right|\right)$, because $b_{k} \rightarrow 0$ (cf. 2.6). Hence (5.2) holds in this case for all $t \in\left(0,\left|a_{j_{1}}\right|\right)$ by $[18,10.12]$. Lemma 2.11 yields

$$
M\left(\Gamma_{t}\right) \leqq\left(\frac{3}{\beta-\alpha}\right)^{n} \int_{C}|\nabla u|^{n} d m,
$$

where $C=R(1, t)$. This estimate together with (5.2) gives the desired lower bound.

5.3. Corollary. Let $u: R^{n} \backslash\{0\} \rightarrow R$ be a bounded monotone $\mathrm{ACL}^{n}$ function and let $\alpha=\liminf \operatorname{in}_{x \rightarrow 0} u(x), \beta=\lim _{\sup _{x \rightarrow 0}} u(x)$. Then

$$
\liminf _{t \rightarrow 0}\left(\int_{R(1, t)}|\nabla u|^{n} d m\right) / \log \frac{1}{t} \geqq c_{n}(\beta-\alpha)^{n},
$$

where $c_{n}$ is the positive constant in the proof of 5.1 .

A counterpart of condition (1.2) for the $\mathrm{ACL}^{n}$ function $u: R^{n} \backslash\{0\} \rightarrow R$ is the following one. There are constants $\mu \in(0,1)$ and $A>0$ such that

$$
\int_{B_{x}}|\nabla u|^{n} d m \leqq A, \quad B_{x}=\bar{B}^{n}(x, \mu|x|)
$$

for all $x \in R^{n} \backslash\{0\}$. From a standard covering argument (cf. [25]) and from (5.4) it follows that

$$
\int_{R(t, t / 2)}|\nabla u|^{n} d m \leqq d(n, A, \mu)
$$

for $t \in(0,1)$, where $d(n, A, \mu)$ depends only on $n, A$ and $\mu$. Furthermore, it follows 
from (5.5) that for $t \in(0,1 / 2)$

$$
\int_{R(1, t)}|\nabla u|^{n} d m \leqq c(n, A, \mu) \log \frac{1}{t} .
$$

A direct calculation shows that the monotone $\mathrm{ACL}^{2}$ function $v(x, y)=y^{2} /\left(x^{2}+y^{2}\right)$, $(x, y) \in R^{2} \backslash\{0\}$ satisfies (5.4) and (5.6), but $v$ fails to have a limit at 0 . This example should be compared with 5.1 .

According to Theorem 5.1 a monotone function with a finite Dirichlet integral has a limit at an isolated singularity. A natural question is whether a similar result holds for a countable sequence of isolated singularities.

5.7. Example. There is a monotone $\mathrm{ACL}^{n}$ function $u: R^{n} \backslash\left\{2^{-k} e_{1}: k=\right.$ $1,2, \ldots\} \backslash\{0\} \rightarrow R$ with

$$
\lim _{x \rightarrow 2^{-k} e_{1}} u(x)=1, \quad \lim _{t \rightarrow 0+} u\left(-t e_{1}\right)=0
$$

$k=1,2, \ldots$ with a finite Dirichlet integral. The existence of such a function $u$ can be seen by a direct construction. Clearly $u$ has no limit at 0 .

The next result is a Phragmén-Lindelöf type theorem.

5.8. Theorem. Let $G \subset R^{n}$ be a domain such that $M\left(R^{n} \backslash G, r, 0\right) \geqq \delta>0$ for all $r \geqq r_{0}$, and let $u: G \rightarrow R$ be a monotone $\mathrm{ACL}^{n}$ function. If

$$
\limsup _{x \rightarrow y} u(x) \leqq 1
$$

for all $y \in \partial G \backslash\{\infty\}$, then either $u(x) \leqq 1$ for all $x \in G$ or

$$
\liminf _{t \rightarrow \infty} \int_{G \cap B^{n}(t)}|\nabla u|^{n} d m / \log t>0 .
$$

Proof. Suppose that $u\left(x_{0}\right)=c>1$ for some $x_{0} \in G$. Let $E=\{x \in G: u(x)<$ $(2+c) / 3\}$. Then $\partial G \subset \bar{E}$ by the assumption. Let $F$ be the $x_{0}$-component of $\{z \in G$ : $u(z)>(1+2 c) / 3\}$. Then $\infty \in \bar{F}$ by 2.6 . Let

$$
\begin{gathered}
\Gamma_{t}=\Delta\left(E, F ; G \cap B^{n}(t)\right), \quad t \geqq r_{0}, \\
\tilde{\Gamma}_{t}=\Delta\left(E, F ; B^{n}(t)\right), \quad t \geqq r_{0} .
\end{gathered}
$$

By the geometry of the situation it follows that $M\left(\Gamma_{t}\right)=M\left(\tilde{\Gamma}_{t}\right) \quad$ (cf. $[18,11.3]$ and (2.10)). From [20,3.5] we obtain

$$
M\left(\tilde{\Gamma}_{t}\right) \geqq c(n, \delta) \log t
$$

for large values of $t$. The proof follows from Lemma 2.11 . 


\section{Some properties of boundary values}

Next we shall compare the limit values of a monotone Dirichlet finite function on the closure of its domain of definition to the limit values on the boundary.

6.1. Theorem. Let $u: R_{+}^{n} \rightarrow R$ be a monotone Dirichlet finite function and let $E \subset \partial R_{+}^{n}$ be a compact set of capacity zero with $0 \in E$. Then

$$
\limsup _{x \rightarrow 0} u(x)=\limsup _{x \rightarrow 0}\left(\limsup _{\substack{y \rightarrow x \\ x \in \partial R_{+}^{n} \backslash E}} u(y)\right) .
$$

Proof. Since cap $E=0$, it follows that $0 \in \overline{\left(\partial R_{+}^{n} \backslash E\right)}$ ([15, p. 72]) and hence the right side of the above equality makes sense. Denote the left and right sides by $\tilde{a}$ and $\tilde{b}$, respectively. Clearly $\tilde{a} \geqq \tilde{b}$. Hence it remains to be shown that $\tilde{a}>\tilde{b}$ is impossible. Choose $a$ and $b$ such that $\tilde{b}<b<a<\tilde{a}$. Let $r>0$ be such that

$$
\limsup _{y \rightarrow x} u(y)<b
$$

for all $x \in\left(\partial R_{+}^{n} \backslash E\right) \cap B^{n}(r)$. Choose a sequence $\left(a_{k}\right)$ in $B_{+}^{n}(r)$ with $u\left(a_{k}\right)>a$ and $\left|a_{k}\right|<r / k$. Let $A_{k}$ be the $a_{k}$-component of the set $\left\{z \in R_{+}^{n}: u(z)>a\right\}$. It follows from 2.6 that $\bar{A}_{k} \cap\left(\partial R_{+}^{n} \cup\{\infty\}\right) \neq \emptyset$ for all $k$. From (6.2) it follows that $\bar{A}_{k} \cap(E \cup$ $\left.\left(\partial R_{+}^{n} \backslash B^{n}(r)\right)\right) \neq \emptyset$ for all $k$. Let $B=\left\{z \in R_{+}^{n}: u(z)<b\right\}$ and $\Gamma_{k}=\Delta\left(A_{k}, B ; R_{+}^{n}\right)$. It follows from 2.11 that

$$
M\left(\Gamma_{k}\right) \leqq(a-b)^{-n} \int_{R_{+}^{n}}|\nabla u|^{n} d m<\infty .
$$

If $\bar{A}_{k} \cap\left(E \cap B^{n}(r)\right) \neq \emptyset$, then $M\left(\Gamma_{k}\right)=\infty$ because $A_{k}$ is a connected set and cap $E=0$ (cf. [18, 10.12]). Otherwise $\bar{A}_{k} \cap\left(\partial R_{+}^{n} \backslash B^{n}(r)\right) \neq \emptyset$, and since cap $E=0$ and $A_{k}$ is connected, we get by $[18,10.12]$ that

$$
M\left(\Gamma_{k}\right) \geqq c_{n} \log k .
$$

In either case we obtain a contradiction with (6.3) when $k \rightarrow \infty$.

6.4. Remark. By inspecting the above proof we see that the condition cap $E=0$ can be weakened. In fact, it suffices to assume that $E \subset \partial R_{+}^{n}$ is a compact set which has no interior points (in the topology of $\left.\partial R_{+}^{n}\right)$ and which satisfies $M\left(y, \partial R_{+}^{n} \backslash E\right)=\infty$ for all $y \in E$ in the sense of [9].

6.5. A bound for a Dirichlet finite function. Let $u: R_{+}^{n} \rightarrow R$ be a monotone Dirichlet finite function, let $E \subset \bar{R}_{+}^{n}$, and let $u$ have a continuous extension, denoted by $u$, to the points $E \cap \partial R_{+}^{n}$ such that $u(x) \leqq b$ for $x \in E$. Define

$$
\sigma(x, E)=\inf _{C} M\left(\Delta\left(C, E ; R_{+}^{n}\right)\right)
$$

where the infimum is taken over all continua $C$ with $x \in C$ and $C \cap\left(\partial R_{+}^{n} \cup\{\infty\}\right) \neq \emptyset$. 
It follows then that

$$
u(x) \leqq b+\left[\left(\int_{R_{+}^{n}}|\nabla u|^{n} d m\right) / \sigma(x, E)\right]^{1 / n}
$$

for all $x \in R_{+}^{n}$. This estimate follows directly from Lemma 2.11 and Remark 2.6. In fact, this idea has been applied several times in this paper. The inequality (6.7) suggests that the quantity $\sigma(x, E)$ is of some interest in the theory of Dirichlet finite functions.

\section{References}

[1] Ahlfors, L. V.: Conformal invariants. Topics in geometric function theory. - McGraw-Hill Book Company, New York et al., 1973.

[2] Beardon, A. F.: The geometry of discrete groups. - Graduate Texts in Mathematics 91, Springer-Verlag, Berlin-Heidelberg-New York, 1983.

[3] Gehring, F. W.: Rings and quasiconformal mappings in space. - Trans. Amer. Math. Soc. 103, 1962, 353-393.

[4] Gehring, F. W., and A. J. Lohwater: On the Lindelöf theorem. - Math. Nachr. 19, 1958, $165-170$.

[5] Gilbarg, D., and N. S. Trudinger: Elliptic partial differential equations of second order. Die Grundlehren der mathematischen Wissenschaften 224, Springer-Verlag, BerlinHeidelberg-New York, 1977.

[6] Granlund, S., P. Lindqvist, and O. Martio: Conformally invariant variational integrals. Trans. Amer. Math. Soc. 277, 1983, 43-73.

[7] Lelong-FerRAND, J.: Représentation conforme et transformations a intégrale de Dirichlet bornée. - Gauthier-Villars, Éditeur-Imprimeur-Libraire, Paris, 1955.

[8] Lelong-Ferrand, J.: Invariants conformes globaux sur les varietes riemanniennes. - J. Differential Geom. 8, 1973, 487-510.

[9] Martio, O.: Equicontinuity theorem with an application to variational integrals. - Duke Math. J. 42, 1975, 569-581.

[10] Mikujukov, V. M.: Boundary properties of a certain class of transformations in space. Trudy Tomsk. Gos. Univ. Ser. Meh.-Mat. 189, 1966, 80-85 (Russian).

[11] Mrkujukov, V. M.: A certain boundary property of $n$-dimensional mappings with bounded distortion. - Mat. Zametki 11, 1972, 159-164 (Russian).

[12] Mostow, G. D.: Quasiconformal mappings in $n$-space and the rigidity of hyperbolic space forms. - Inst. Hautes Études Sci. Publ. Math. 34, 1968, 53-104.

[13] Oнтsuka, M.: Dirichlet problem, extremal length and prime ends. - Van Nostrand Reinhold Company, New York-Cincinnati-Toronto-London-Melbourne, 1970.

[14] RešETNJAK, Ju. G.: The concept of capacity in the theory of functions with generalized derivatives. - Sibirsk. Mat. Ž. 10, 1969, 1109-1138 (Russian).

[15] ReŠETNJAK, JU. G.: Spatial mappings with bounded distortion. - Izdatel'stvo Nauka, Novosibirsk, 1982 (Russian).

[16] Rickman, S.: Asymptotic values and angular limits of quasiregular mappings of a ball. Ann. Acad. Sci. Fenn. Ser. A. I. Math. 5, 1980, 185-196.

[17] Suvorov, G. D.: The metric theory of prime ends and boundary properties of plane mappings with bounded Dirichlet integrals. - "Naukova Dumka", Kiev, 1981 (Russian). 
[18] V̈̈ISÄLÄ, J.: Lectures on n-dimensional quasiconformal mappings. - Lecture Notes in Mathematics 229, Springer-Verlag, Berlin-Heidelberg-New York, 1971.

[19] V̈̈ISÄLÄ, J. : A survey of quasiregular maps in $R^{n}$. - Proceedings of the International Congress of Mathematicians, Helsinki 1978, 685-691.

[20] VuORINEN, M.: Lower bounds for the moduli of path families with applications to non-tangential limits of quasiconformal mappings. - Ann. Acad. Sci. Fenn. Ser. A. I. Math. 4, 1978/79, 279-291.

[21] Vuorinen, M.: On the existence of angular limits of $n$-dimensional quasiconformal mappings. Ark. Math. 18: 2, 1980, 157-180.

[22] Vuorinen, M.: Capacity densities and angular limits of quasiregular mappings. - Trans. Amer. Math. Soc. 263, 1981, 343-354.

[23] Vuorinen, M.: On the Harnack constant and the boundary behavior of Harnack functions. Ann. Acad. Sci. Fenn. Ser. A. I. Math. 7, 1982, 259-277.

[24] Vuorinen, M.: On the uniqueness of sequential limits of quasiconformal mappings. - Math. Scand. 52, 1983, 69-80.

[25] Vuorinen, M.: The boundary behavior of quasiregular mappings. - In preparation.

[26] Ziemer, W. P.: Extremal length and p-capacity. - Michigan Math. J. 16, 1969, 43-51.

University of Helsinki

Department of Mathematics

SF-00100 Helsinki 10

Finland

Received 13 March 1984 